\title{
Methods for combined treatment of oil- containing waste water of transportation enterprises
}

\author{
Irina Okhremenko, and Abdumalik Rizaev ${ }^{1 *}$ \\ Tashkent State Transport University, Tashkent, Uzbekistan
}

\begin{abstract}
The article discusses modern schemes for the treatment of oily wastewater from transport enterprises. The features of closed water supply systems and the fundamental provisions for the design of a compact module for wastewater treatment of transport enterprises are revealed.
\end{abstract}

\section{Introduction}

Protecting water resources from depletion and pollution is one of the most important environmental problems. Oil products in industrial discharges are represented by various components:

- in the form of a floating film

- in emulsion form

- dissolved forms.

The removal of each of the above groups from wastewater occurs by a certain method, and the greatest cleaning effect is achieved by their combination. Selecting the optimal process equipment requires a thorough analysis of a specific flow.

- Are important:

- consumption of the liquid to be cleaned

- initial content of oil products

- cleaning standards

Vehicles carry the bulk of oil pollutants into wastewater. The presence of a circulating water system is one of the most important indicators of the technical level of industrial enterprises. Therefore, the task of developing treatment facilities is an urgent problem of resource conservation. The aim of the work was to develop an integrated technology for the purification of oil and oily wastewater from transport enterprises and disposal of sediments, depending on their physical and chemical composition

*Corresponding author: mr.rizaev.58@mail.ru 


\section{Method}

When choosing methods and technologies for treatment applicable to specific wastewaters, the main predetermining parameters are composition, quantity, and mode of production water inflow at transport enterprises. These parameters are different and depend on the type of enterprises and technological processes at them. The amount of generated industrial effluents is relatively small. Having data on wastewater consumption, their detailed characteristics, including the content of impurities, as well as requirements for treated water, several methods are selected for verification. On the basis of experimental studies, taking into account technical and economic indicators, the optimal method is selected. The choice depends on many factors: the amount of various types of wastewater, their consumption, the possibility and economic feasibility of extracting the detected harmful impurities; requirements for the quality of purified water when it is used for repeated and recycling water supply and discharge into a reservoir, the capacity of a reservoir, the presence of district or city treatment facilities.[4]

The following composition of wastewater is typical for railway transport enterprises. The concentration of oil products in wastewater is from 500 to 30,000 $\mathrm{mg} / 1$. Existing methods of purification make it possible to bring their concentration to $50-300 \mathrm{mg} / \mathrm{l}$, which does not ensure the possibility of reusing water for technological needs, and oil and oil products released as a result of purification are currently sent to oil sludge storage.

Petroleum products in wastewater are most often found in three main states:

- in molecularly dissolved, particle size 10-5>d $>10-7 \mathrm{~m}$;

- emulsified, particle size 10-3>d $>10-5 \mathrm{~m}$;

- dispersed, with particle size $\mathrm{d}>10-3 \mathrm{~m}$.

The technical audit of the state of treatment facilities at the enterprises of the company "Uzbekiston Temir Yulari" revealed that the existing technologies for treating waste water at the enterprises of railway transport of the Republic of Uzbekistan are imperfect. They are distinguished by the high cost of cleaning, low productivity, high consumption of electricity, water, and steam. An analysis of the operation of treatment facilities existing at railway transport enterprises shows that the currently used methods and technological schemes for the purification of oily wastewater, mainly sedimentation, most often do not ensure a decrease in the content of harmful substances to concentrations that allow water to be reused or sent to the city sewage system or dump into a body of water. A significant number of oil sludge and waste storage facilities, built since the early $1950 \mathrm{~s}$, have evolved from a means of preventing oil pollution to a permanent source of such pollution. Volley discharges into the urban network have a disastrous effect on biological treatment facilities. Due to the actual absence of a cleaning solution regeneration unit, such a valuable product as caustic soda is lost.

The objects of research are highly concentrated wastewater from the enterprises of UE "Uztemiryulmashtamir," Wagon depot No. 2, VChD-2. Waste waters from these enterprises were examined.

Below in (table 1.), The results of the analysis of wastewater from enterprises of UE "Uztemiryulmashtamir", Wagon Depot No. 2, VChD-2 for the content of oil products (fuels and lubricants, fuels and lubricants) and fats are given. This is wastewater from cleaning and repairing locomotives, passenger cars, train preparation service. 
Table 1. Indicators of wastewater composition of UE "Uztemiryulmashtamir,

"Wagon depot No. 2, VChD-2

\begin{tabular}{|c|c|c|}
\hline \multirow{2}{*}{ Dates } & \multicolumn{2}{|c|}{ Quantity, mg/l } \\
\cline { 2 - 3 } & \multirow{2}{*}{$\begin{array}{c}\text { Fuels and lubricants } \\
\text { (petroleum products) }\end{array}$} & Oils(fats) \\
\cline { 1 - 3 } UE "Uztemiryulmashtamir" & 26.5 & - \\
\hline 28.05 .2002 & 5.86 & 5.36 \\
\hline 12.06 .2002 & 3.3 & 2.5 \\
\hline 12.12 .2005 & 6.57 & 16.45 \\
\hline 26.12 .2005 & 2.14 & 4.28 \\
\hline 29.05 .2006 & 1.23 & 4.16 \\
\hline 16.10 .2006 & 7.47 & 5.18 \\
\hline 07.06 .2007 & & 4.16 \\
\hline Wag depot №2 & 1.23 & 3.13 \\
\hline 16.10.2006 & 1.38 & 5.0 \\
\hline VCHD-2 & & \\
\hline Ecological MPC & 1.0 & \\
\hline & &
\end{tabular}

As can be seen from the above data, the content of oil products in wastewater discharged into the sewage system is high - the MPC exceeds up to 8 times, and fats - more than 4 times. Oil sludge arising from wastewater processing refers to tank-type oil sludge.

The most effective measure to reduce the negative impact of railway facilities on the environment is the creation of recycling water supply and wastewater treatment systems carried out at local treatment facilities without discharging them into surface water bodies, municipal and storm sewers.

Properties of the pollutants they contain. So, first of all, it is not taken into account that oily wastewater is a multicomponent and multiphase water system.

The choice of the variant of the technological scheme is made, taking into account the real quality indicators of oily waters to be treated, wastewater consumption, and requirements for the quality of the treated water. Long-term practice shows that reliable, high-quality purification of oily waters is possible only when implementing multistage technological schemes for extracting oil products and other polluting ingredients. Attempts to use simple solutions for obtaining purified water of the required quality give only a short-term effect and are not suitable for the long-term operation of treatment facilities.

When choosing a technology for treating a particular runoff, the determining factors are runoff flow rate, initial concentration of oil products and associated pollution, requirements for the quality of treated water for all standardized pollution. Usually, cleaning is carried out in several stages, each of which ensures removing oil products from the effluent, which are in a certain phase-dispersed state. As a rule, such multistage schemes consist of a stage of pretreatment from non-emulsified and coarse-emulsified oil products, a stage of the main purification from fine emulsified particles, and a stage of additional purification from dissolved oil products. The treatment of such waters includes the following stages: sedimentation, reagent treatment, flotation, filtration. Any wastewater treatment to the required degree is achieved by creating a multi-level complex consisting of the above treatment plant systems.

At the first stage, cleaning, as a rule, is carried out in oil traps equipped with mechanisms for collecting and removing a layer of captured oil products. Recently, due to the presence of suspended solids in almost all oily waters, combined installations - oil traps - are widely used. Alternatively, it is possible to replace them with three-product hydrocyclones, which are especially beneficial when space is limited for the placement of treatment facilities. The second cleaning stage consists mainly of two stages. The first stage is designed to extract the bulk of finely emulsified oil products and highly dispersed and 
colloidal solid impurities. Most often, settling tanks or flotation with preliminary coagulation treatment of effluents are used for cleaning at this stage. In this case, either chemical coagulation with reagents or electrocoagulation in electrolyzers with soluble aluminum or steel electrodes is performed. The galvanic coagulation method of processing oily waters before settling is also gaining popularity. But this method of introducing a coagulant into the treated effluent can only be used with their high mineralization or acidity $(\mathrm{pH}<2)$.

The disadvantages of these installations are low efficiency and cumbersome design. In order for the wastewater to be cleaned from the suspension of oil products to the required degree, the residence time of the water in the sump should be several hours. For effective cleaning, the dimensions of the oil and petrol separator must also be significant.

The solution to all these issues lies in developing compact treatment facilities and the transition to a closed system of technological water use, which would be not only ecologically justified but also economically justified. Purely mechanical methods of water purification from oil products are of limited use and ineffective. Therefore, in modern technology for the treatment of oily wastewater, schemes and installations are used, where mechanical, physicochemical, and chemical methods are combined in combination.

The modern treatment plant market offers a wide range of equipment for the treatment of oily wastewater. Purification of small amounts of wastewater (from 1,5 to $100 \mathrm{~m} 3 / \mathrm{h}$ ), generated at oil depots, warehouses of fuels and lubricants, auto enterprises, railway stations is usually carried out at prefabricated plants [1]. The units include various combined apparatuses, including sedimentation, coagulation, flotation, sorption in one tank, and column-type structures for mechanical, physicochemical, and biological treatment. The combined facility for treating industrial and industrial storm wastewater of various categories allows for mechanical treatment in a whirlpool and a sump, physical and chemical treatment using electromechanical coalescers of various modifications with soluble and insoluble electrodes and separation of coagulated flakes of contaminants in thin-layer blocks and a filter-settler.

The most promising is the use of flotation and membrane methods, which have several advantages: simplification of the technological scheme, ease of automation, reduction of production areas, reduction of the amount of precipitation [2].

\section{Results and Discussion}

For transport enterprises, locomotive and carriage depots, gas stations, washes of various types of production equipment, vehicles and rolling stock, whose wastewater contains oil products classified as highly hazardous and strictly regulated by the maximum permissible concentration (MPC) standards, by specialists of the Tashkent State University a compact modular water purification unit has been developed.

The compact module designed at the Department of Engineering Communications and Systems is a combined structure consisting of circular containers in the form of hydrocyclones. The designed plant consists of four off-center cylinders. By design, the tanks of the compact module are: the first cylinder - a pressure hydrocyclone with a downward flow; a thin-layer sedimentation block located in the lower part of the second cylinder - a thin-layer multi-layer hydrocyclone formed by coaxially arranged plates; the second cylinder plays the role of a clarifier in the suspended layer, where in the cylindrical part of the apparatus, with a decrease in the velocity of the ascending flow to $1 \mathrm{~mm} / \mathrm{s}$, the effect of the gravitational field increases. Here filtration takes place through a suspended layer of sediment, provided that the flow continues to rotate, which also implies the separation of the suspension due to centrifugal forces. The third cylinder is a downdraft hydrocyclone combined with a flotation device, the counterflow of air bubbles from the air- 
water mixture supplied here facilitates the separation and flotation of fine particles of impurities; the fourth cylinder serves as a shell of the sorption filter.

Mechanical cleaning: In 1-stage, oily waste water, pre-mixed with reagents, is supplied to the hydrocyclone under pressure through a feed nozzle installed tangentially directly under cover of the apparatus. Waste water is discharged through the nozzle, and the drain is through a drain pipe located in the center of the module and directly connected to the drain pipe. The centrifugal force arising from the rotation of the pulp due to the tangential feeding of the feed drives the particles out of the flow to the outer wall of the cyclone. Since the rate of radial migration of particles is proportional to the density of particles and their diameter squared, the larger and heavier particles have time to leave the flow core, while the smaller ones, for the most part, remain in the flow core. As a result, a large fraction of particles is discharged through the sludge nozzle. With the vortex motion of the liquid in the hydrocyclone, two rotating flows are formed - an external one, moving along the walls of the cone down to the slurry nozzle, and an internal cylindrical one directed upwards along the axis to the drain pipe. Near the geometrical axis of the apparatus, the centrifugal force becomes so great that a rupture of the liquid occurs - an air core (air column) is formed around the axis. Its diameter is $0.6-0.7$ times the diameter of the drain pipe $d$. The tangential velocity of the pulp increases with decreasing distance from the axis; therefore, a sharp increase in centrifugal force from the walls to the axis is observed in the hydrocyclone. To describe the flow field in a hydrocyclone, two-dimensional axisymmetric Reynolds equations are used, which are best suited for describing an axisymmetric flow regime [11]

The axial velocity in the external flow is directed downward and in the internal one upward. Then the flow changes the direction of movement and, heading upwards, enters a thin-layer multi-hydrocyclone.

2-stage - a block of thin-layer sedimentation, formed by coaxially located plates dividing the flow into thin layers, provides for a laminar flow regime, but here the centrifugal force field is still strongly applied to retain coarse and emulsified oil particles, as well as mineral impurities. At this stage, the removal of suspended particles by $40-50 \%$ is achieved. The thin-layer sedimentation block is designed as a multi-tiered hydrocyclone.

According to the analysis of the kinetic curves of wastewater sedimentation at a temperature of $20^{\circ} \mathrm{C}$, in a layer of $\mathrm{h}=200 \mathrm{~mm}$, the required degree of purification is achieved when particles with a particle size of $0.2 \mathrm{~mm} / \mathrm{s}$ are retained.

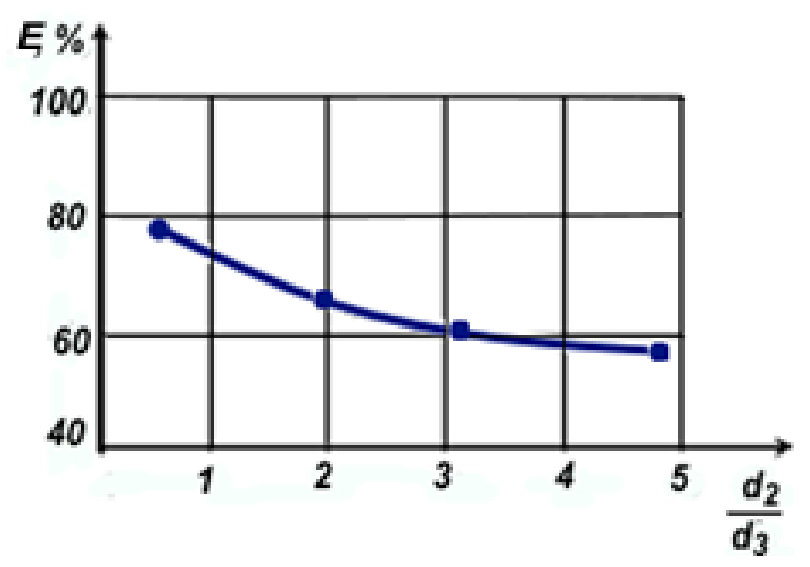

Fig. 1. Experimental plot of suspension removal kinetics 
Flotation takes place in two stages. The action of a pressure flotation device is based on the ability of air to dissolve in water at elevated pressure. When the pressure is released, the dissolved air is released in the form of tiny bubbles, which, floating up, capture and carry contamination particles to the surface. Floating takes place directly in the flotator and the hydrocyclone following it, where, once again acquiring rotational motion, it encounters a counterflow of air bubbles from the water-air mixture supplied here, which contributes to the separation and flotation of fine particles of contaminants. The separated suspension containing the dissolved gas is fed into the casing through the branch pipe and moves in the film flow mode along a spiral trajectory along the inner surface of the casing wall, made in the form of a screw surface of a sinusoidal profile, the direction of cutting of which coincides with the direction of rotation of the flow of the separated suspension, the distance between the protrusions of which increases, and the amplitude a decrease in the axial direction, which makes it possible to increase the thickness of the film and the residence time of the separated suspension in the hydrocyclone, especially in the zone of the most intense release of gas bubbles and the formation of flotation complexes near the inlet. The proposed design of the hydrocyclone-flotator allows to reduce the attenuation of the circumferential component of the speed of the separated suspension in the direction of the hydrocyclone axis and to increase the filling of the profile of the radial distribution of the circumferential component of the speed by creating a near-wall gas layer with low viscosity, to increase the kinetic coefficient of flotation and the speed of transporting flotation complexes to the surface of the suspension film, also increase the number of formed flotation complexes by increasing the film thickness and the residence time of the separated suspension in the hydrocyclone, as a result of which the separation capacity.

In the conical part of the hydrocyclone-flotator, the flow again changes its direction of movement and, moving upward, enters the sorption filter. Here, finely dispersed particles of petroleum products undergo an additional cleaning process by filtration through a sorbent. Depending on the required degree of purification, the sorption filter can have one or two stages.

Filters of the 1st stage are used to purify drains that have undergone mechanical treatment and flotation from emulsified oil products, oils, and resins. The original design of the filter allows the use of various types of filter material (crushed expanded clay, slags, polyurethane, etc.) as a load, but the most effective was an activated aluminosilicate adsorbent made of natural clay with activating additives. The source water was taken at the local treatment facilities of the enterprise. The experiments were carried out on initial water at filtration rates of 3,4 , and $5 \mathrm{~m} / \mathrm{h}$, three series of experiments for each speed, where the maximum degree of purification in most cases was no less than $89 \%$. Based on the results obtained, it can be concluded that the activated aluminosilicate adsorbent gives a fairly high percentage of wastewater treatment from oil products (Fig. 2 and Fig. 3). [7] High adsorption, catalytic, ion-exchange properties of clay minerals, the availability of effective methods for regulating their geometric structure and the chemical nature of the surface, the existence of large industrial deposits, and the low cost of clays make it economically feasible to use them in Uzbekistan. 


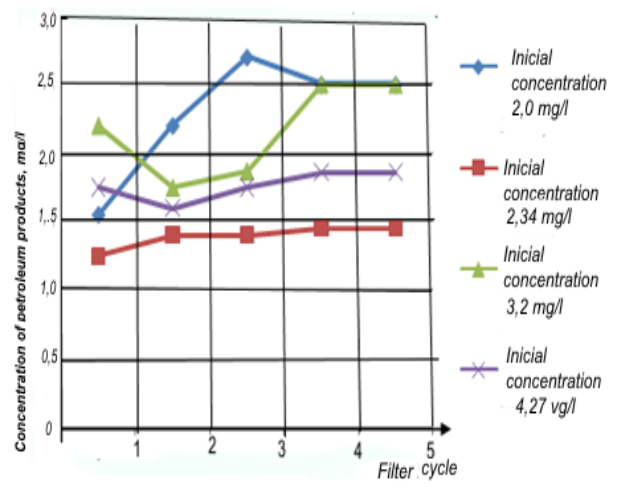

$\mathrm{V}_{\text {max.f }}=4 \mathrm{~m} / \mathrm{h}$

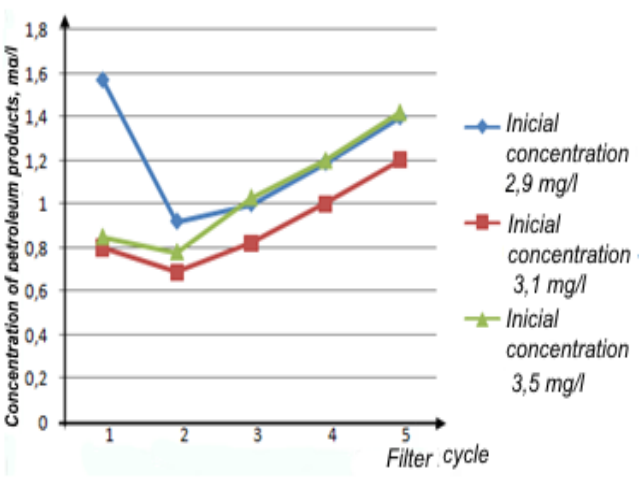

$\mathrm{V}_{\text {max.f }}=3 \mathrm{~m} / \mathrm{h}$

Fig. 2. Dynamics of additional treatment of the real runoff of TashTVRZ and VChD-2 from oil products on a short layer of activated aluminosilicate adsorbent
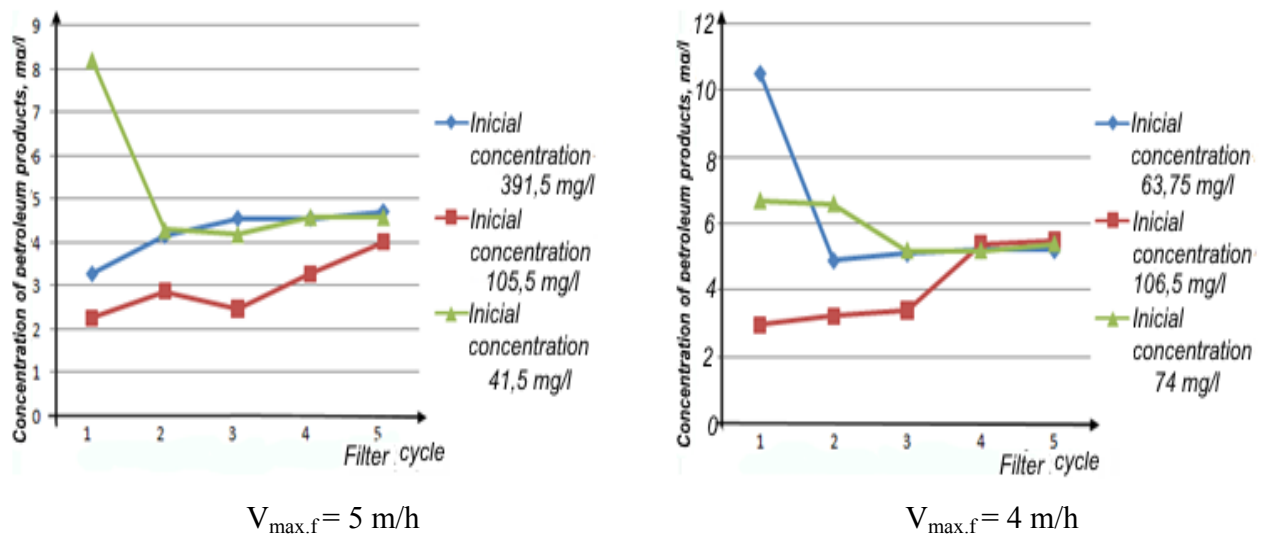

Fig. 3. Dynamics of treatment of concentrated wastewater from a locomotive depot from oil products

The regimes of the treatment plant recommended by the developer: filtration rate 5-7 $\mathrm{m} / \mathrm{h}$; washing intensity $14-15 \mathrm{l} / \mathrm{s}^{*} \mathrm{~m}^{2}$; water consumption for flushing - three volumes of adsorbent. Expansion of the adsorbent during flushing - 30\%. The recommended height of the adsorbent layer in the filter is $1.2-1.7 \mathrm{~m}$. The frequency of activation is after $3-5$ or more filter cycles. The circulation rate of the activator solution is $2-31 / \mathrm{s}^{*} \mathrm{~m}^{2}$. The hydrogen index of the filtrate $(\mathrm{pH})$ is 7.5-9.0. The concentration of pollutants in the filtrate is practically independent of the initial concentration of contaminants. If shock doses of contaminants enter the sorption filters, the filter cycle is reduced, while the excess of MPC is not observed. It is also noted that exceeding the filtration rate significantly reduces the degree of wastewater treatment. The loss of adsorbent for abrasion is $5-10 \%$ per year.

Sorption filters are washed once a day with filtrate from the storage tank. Washing one filter requires $30-100 \mathrm{~m} 3$ of water, washing time -6 minutes. The adsorbent is activated once a month with a $7 \%$ solution of soda ash.

The spent filter material and flotation sludge are fed to the sludge processing complex. The sludge discharged as a result of cleaning the enterprises of the Uzbekiston Temir Yllari SJCK is sent to the sludge storage facilities. They contain heavy and medium fractions of 
petroleum products, heavy oils, mechanical impurities, and ions of sodium, potassium, calcium, copper, chromium, nickel. Oil sludge arising from wastewater processing refers to tank-type oil sludge.

Studies have established that the mass loss of the sludge during heat treatment begins at a temperature of $100^{\circ} \mathrm{C}$, ends completely at a temperature of $600^{\circ} \mathrm{C}$, and further heating does not lead to a loss of mass. Weight loss occurs due to the removal of hygroscopic moisture and moisture bound in chemical compounds, as well as due to the burnout of organic compounds and decomposition of sulfates. Ash contains a fixed form of heavy metals. The temperatures of endothermic effects and sludge decomposition were determined (Fig. 4). The kinetics of this process was studied to determine the optimal time for heat treatment of the sludge. Three samples were heated in parallel to a temperature of $600^{\circ} \mathrm{C}$. In this case, the change in the mass of the samples was recorded. (Fig. 5). The above results indicate that a stable state of the sediment is achieved after $2 \mathrm{~h}$ of heat treatment.

Cleaning and disposing of oil sludge. The advantages of this method are the adsorption of substances in multicomponent mixtures and high purification efficiency. Based on the conducted studies of the physical and chemical composition of highly concentrated waste water from transport enterprises, to utilize this waste, a technology for producing a sealing lubricant has been developed.

Studies to determine the operational properties of the developed sealing grease using the antifriction additive of the spent sorbent STRG showed that the filler regulates the surface properties - lubricating and sealing ability, protective properties, as well as stability.

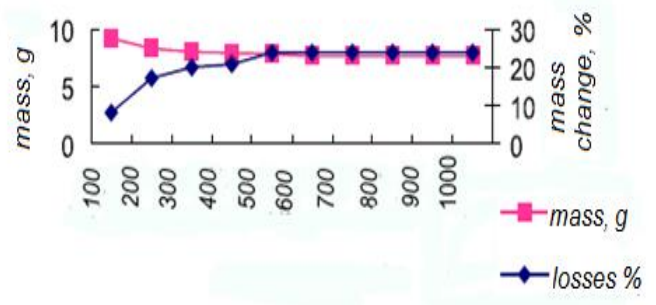

Fig. 4. Indicators of sludge heat treatment

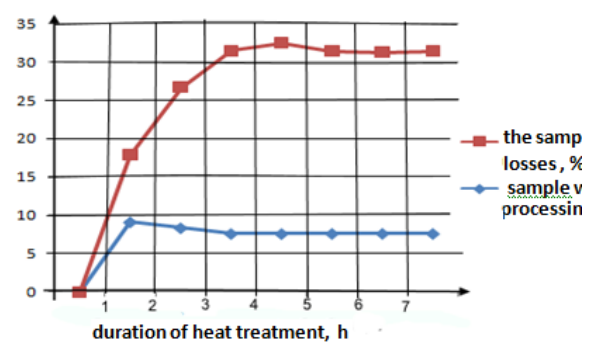

Fig. 5. Kinetics of sludge heat treatment

The presented design solution for an oily wastewater treatment plant and oil sludge processing differs from traditional solutions for using oil traps. The volume and dimensions of the module and its individual elements are calculated in relation to local conditions. Level sensors are placed in the receiving tank, which, depending on the volume of wastewater and the mode of their inflow, switch the unit on and off. Installation productivity $-2 \div 20 \mathrm{~m} 3 / \mathrm{h}$; dimensions $-\mathrm{D}=1.5 \div 3.5 \mathrm{~m}, \mathrm{H}=2.5 \div 4.5 \mathrm{~m}$; purification degree - for oils and petroleum products - 95-98\%, for suspended solids $-80-95 \%$.

\section{Conclusions}

1. The disadvantages of traditional methods of water purification of transport enterprises from oil products are high cost, low productivity, high consumption of energy, water and steam, the need for large-scale treatment facilities or expensive equipment for separating oil products

2. The developed compact modular installation makes it possible to significantly reduce the territory of treatment facilities, as well as the costs of their operation and fines for pollution of the environment and urban wastewater. 
3. According to the analysis of the kinetic curves of wastewater sedimentation at a temperature of $20{ }^{\circ} \mathrm{C}$, in the layer $\mathrm{h}=200 \mathrm{~mm}$, the required degree of purification is ensured when particles with a particle size of $0.2 \mathrm{~mm} / \mathrm{s}$ are retained.

4. In the sorption post-treatment unit, the concentrations are $1 \div 5 \mathrm{mg} / \mathrm{l}$, according to calculations and experiments, the required filtration mode is carried out at the lowest possible speeds in the range from 2 to $3 \mathrm{~m} / \mathrm{h}$.

The most effective adsorption method has been selected for cleaning and utilizing oil sludge. The advantages of this method are the adsorption of substances of multicomponent mixtures and high purification efficiency.

\section{References}

1. Valley L.F. Modern technology and facilities for the treatment of oily wastewater, Monograph. - Dnepropetrovsk: Continent,.p. 296. (2005)

2. Pavlov D. V., Varaksin S. O., Kolesnikov V. A., Vasiliev R. N. Universal technology of wastewater treatment from oil products - Sanitary engineering " 3, pp. 28-32 (2011).

3. Rizaev A. Research methods of water purification from pollution with petroleum and petroleum products - International Journal of Psychosocial Rehabilitation. 24, (08), (2020)

4. Matveeva, KA Treatment of oily waste water from the motor transport industry using a closed water supply system, Young scientist. 8 (298). pp. 39-44. URL. (2020)

5. Rizaev A.N., Okhremenko I.M. Treatment of oily waste water from transport enterprises: Monograph. - Tashkent: LLC "ADAD PLYUS", p. 167. (2018).

6. Okhremenko I.M. Ergashev Sh.Sh. Resource-saving technology of wastewater treatment of enterprises of JSC "Uzbekiston Temir Yulari", Proceedings of the republican scientific and technical conference "Resource-saving technologies in railway transport" -T. TashIIT, (2017)

7. Rizaev A.N., Okhremenko I.M. Module for complex treatment of oily wastewater from railway transport enterprises - "Akhborot". 2. (2018)

8. Ergashev Sh.Sh. Assessment of the properties of sorbents used in wastewater treatment. Materials of the fourth academic readings, RAASN, St. Petersburg, PGUPS, March 19-20,. pp. 111-114. (2009)

9. Ivanov V. G., Ergashev Sh. Sh. Research and development of new technical devices for wastewater treatment of railway transport enterprises - Scientific electronic library "CyberLeninka"

10. Kapustin R.P. Determination of the velocities of fluid movement in hydrocyclone devices. Sat. proceedings of the 4th International Scientific and Technical Conference. Bryansk., pp. 39-41. (2001).

11. Medvedeva V.M. Technology of deep purification of oily waste water from railway transport facilities using an activated aluminosilicate sorbent, Dissertation for the degree of Ph.D. (2008).

12. Rizaev A.N. Shamansurov S.S. Technology of purification and utilization of oil sludge from railway transport production, Materials of the International scientificpractical conference "Oil refining 2008". (Russia) Ufa, (2008)

13. Chernenko T.V., Imatullina G.K., Rezuanov D.A., Kireeva A.T., Khalitov A.K. Methods for treating wastewater from oil product, Problems and prospects of modern science, collection of scientific papers of the Fourth International Teleconference "Fundamental Sciences and Practice". 3 (1). - Tomsk - (2011). 
14. Anapolskiy V.N., Prokopyev K.L., OliferK S.V.,. Romanenko A.P., Actual problems of oily wastewater treatment - Journal of Plumbing, Heating, Air Conditioning June (2007)

15. Zheltobryukhov V. F., Feldshtein E. G. About methods of cleaning surface runoff of motor transport enterprises - Scientific electronic library "CyberLeninka"

16. Nesterov A.V, Purification of oily waste water by a combination of extraction and adsorption methods, Dissertation for the degree of Ph.D. - Ivanovo: IGKhTU, (2008).

17. Strepetov I.V. Development of a technology for the treatment of oily wastewater using a mixed reagent, Dissertation for the degree of Ph.D. - Volgograd: VGISU, (2012)

18. Labunska I. Russian Refuse III. Investigation of organic and heavy metal contaminants input and distribution in selected rivers of the Russian Federation, Iryna Labunska, Kevin Brigden, David Santillo, Alexey Kiselev, Paul Johnston// Greenpeace Research Laboratories Technical Note 04. pp. 14 - 18. (2011)

19. Grützmacher G, Bank filtration and aquifer recharge for drinking water production, application, efficiency and perspectives - an integration of NASRI outcomes and international experiences/ G. Grützmacher, B. Wiese, I. Hülshoff, D. Orlikowski, E. Hoa and Y. Moreau-Le Golvan/ Kompetenzzentrum Wasser Berlin gGmbH - Berlin, Germany,. pp. 3-4. (2012).

20. Khamidov B.N., Toshtemirov B.V., Ubaydullaev B.Kh., Khosilov Kh.N. Development of a new technology for producing axle oil for railway transport / Uzbek Journal of Oil and Gasб № 3, pp. 35-37, Tashkent, (2011) 